\title{
Exploring Transient, Neutronic, Reduced-Order Models using DMD/POD-Galerkin and Data-Driven DMD
}

\author{
Rabab Elzohery and Jeremy Roberts \\ Department of Mechanical and Nuclear Engineering, Kansas State University \\ 3002 Rathbone Hall, Manhattan, KS 66506, US \\ rababelzohery@ksu.edu,jaroberts@ksu.edu
}

\begin{abstract}
There is growing interest in the development of transient, multiphysics models for nuclear reactors and analysis of uncertainties in those models. Reduced-order models (ROMs) provide a computationally cheaper alternative to compute uncertainties. However, the application of ROMs to transient systems remains a challenging task. Here, a 1-D, twogroup, time-dependent, diffusion model was used to explore the potential of three different ROMs: the intrusive POD-Galerkin and DMD-Galerkin methods and the purely datadriven DMD. For the problem studied, POD-Galerkin exhibited by far the best accuracy and was selected for further application to uncertainty propagation. Perturbations were introduced to the initial condition and to the cross-section data. A greedy-POD sampling procedure was used to construct a reduced space that captured much of the variation in the uncertain these parameters. Results indicate that relatively few samples of the uncertain parameters are needed to produce a basis for POD-Galerkin that leads to distributions of the quantities of interest that match well with those obtained from the full-order model using brute-force, forward sampling.
\end{abstract}

KEYWORDS: ROM, UQ, Galerkin projection, DMD

\section{INTRODUCTION}

A series of transient neutronic experiments has been performed at the University of Wisconsin Research Reactor (UWNR). The evaluation of these experiments involves the quantification of uncertainties in some responses of interest due to those in some input parameters. Monte-Carlo sampling enjoys several advantages as it can capture non-linear effects besides it is suitable for high dimensional parameters space. However it remains computationally challenging since it demands a large number of model executions to ensure convergence. As an alternative, a reduced-order model $(\mathrm{ROM})$ that well-approximates the full-order model (FOM) can be used to propagate uncertainties.

Reduced-order models can be broadly classified as intrusive and non-intrusive. Non-intrusive methods are data-driven, i.e., the FOM is a black box that used solely to generate data. Such methods include Gaussian processes (GPs) and dynamic mode decomposition (DMD), which previously been applied in the nuclear-analysis domain [1], [2], [3], [4]. Contrarily, intrusive methods require modification of the governing equations and, thus, require direct access to the source code. Projection-based methods, including Galerkin and related schemes, are the most common of these methods and have also been used to construct neutronic ROMs [5], [6]. 
The present work aims to illustrate the potential utility of two intrusive ROMs (one based on Galerkin proper-orthogonal decompositon (POD) and one based on DMD-Galerkin) and one nonintrusive ROM (based on DMD) for transient neutronic modeling and uncertainty quantification. To facilitate implementation of the intrusive methods, a simple, 1-D transient was modeled based on two-group diffusion with 8 precursor groups. All three ROMs were tested, and of these, PODGalerkin demonstrated the best accuracy and, hence, this ROM was further explored for uncertainty propagation. Projection-based ROMs require a set of basis function onto which to project the solution. To maximize the accuracy of POD-Galerkin for a variable parameter space (e.g., uncertain cross sections and control positions), a greedy sampling technique [7], [8] was used to select the most suitable snapshots from the black-box model.

\section{Model Problem}

The model problem used is a slah reactor, as shown in Figure 1, where $\mathrm{R}$ indicates a reflector and $A_{i}$ specifies the $i$ th assembly. All of the assemblies are of the same fuel type, but $A_{2}$ and $A_{4}$ are control locations. Listed in Table 1 are the two-group constants for the reflector, fuel with no control inserted, and fuel with control fully inserted. Linear interpolation is used to compute $\Sigma_{a 2}$ value at intermediate control positions. Precursor data are shown in Table 2.

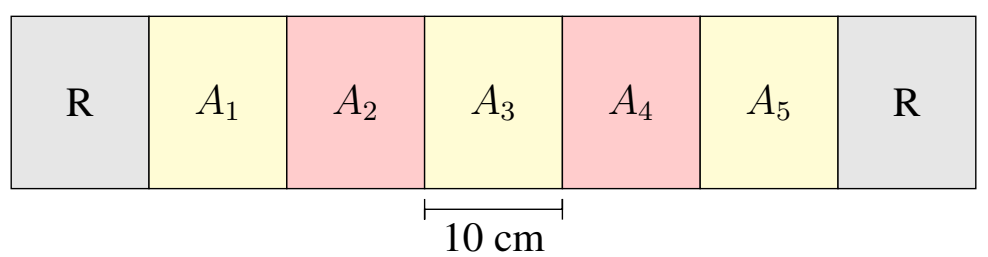

Figure 1: 1-D reactor model

The transient begins in steady state with both control elements $25 \%$ withdrawn. After 2 seconds, the right control (in $A_{4}$ ) is removed to $30 \%$ withdrawn over a period of 2 seconds at a constant rate. This configuration is maintained for 6 seconds. At 10 seconds, the right control is inserted to the $25 \%$ position over a period of 2 seconds at a constant rate. The reactor power is followed for an additional 50 seconds, for a total of 62 seconds.

To solve this problem numerically, a mesh-centered, finite-difference discretization was used in space. The discretized system can be described in the compact form $d \mathbf{y} / d t=\mathbf{A}(t) \mathbf{y}$, where $\mathbf{y}$ represents all responses of interest, i.e., two-group fluxes and precursor concentrations, and $\mathbf{A}$ is the time-evolution matrix, which contains neutron and precursor removal and production terms. Because of the control rod movement, the absorption cross-section changes with time, and, hence, $\mathbf{A}$ is time dependent. For the time discretization, the backward-Euler method was used, leading to

$$
\mathbf{y}^{k+1}(\mathbf{I}-\mathbf{A} \Delta t)=\mathbf{y}^{k}
$$

where $k$ denotes the time step and $\Delta t$ is the time step size. A spatial step of $0.2 \mathrm{~cm}$ and temporal step of $0.1 \mathrm{~s}$ were used for all calculations. 


\begin{tabular}{|c|c|c|c|c|c|c|c|}
\hline material & $D_{1}$ & $D_{2}$ & $\Sigma_{a 1}$ & $\Sigma_{a 2}$ & $\Sigma_{s 2 \leftarrow 1}$ & $\nu \Sigma_{f 1}$ & $\nu \Sigma_{f 2}$ \\
\hline reflector & 1.5000 & 0.5000 & 0.0002 & 0.0100 & 0.0320 & 0.0000 & 0.0000 \\
\hline fuel (all out) & 1.3000 & 0.5000 & 0.0105 & 0.1140 & 0.0220 & 0.0030 & 0.1900 \\
\hline fuel (all in) & 1.3000 & 0.5000 & 0.0105 & 0.1640 & 0.0220 & 0.0030 & 0.1900 \\
\hline
\end{tabular}

Table 1: Two-group diffusion constants

\begin{tabular}{|c|c|c|c|c|c|c|c|c|}
\hline group $i$ & 1 & 2 & 3 & 4 & 5 & 6 & 7 & 8 \\
\hline$\beta_{i}$ & $2.18 \mathrm{e}-04$ & $1.02 \mathrm{e}-03$ & $6.05 \mathrm{e}-04$ & $1.31 \mathrm{e}-03$ & $2.20 \mathrm{e}-03$ & $6.00 \mathrm{e}-04$ & $5.40 \mathrm{e}-04$ & $1.52 \mathrm{e}-04$ \\
\hline$\lambda_{i}$ & $1.2467 \mathrm{e}-02$ & $2.8292 \mathrm{e}-02$ & $4.2524 \mathrm{e}-02$ & $1.33042 \mathrm{e}-01$ & $2.92467 \mathrm{e}-01$ & $6.66488 \mathrm{e}-01$ & 1.634781 & 3.554601 \\
\hline
\end{tabular}

Table 2: Eight-group, delayed-neutron precursor constants

\section{Surrogate Models Construction}

In this section, three different reduced order models are presented. The methods include two Galerkin projection based methods, i.e, POD-Galerkin and DMD-Galerkin, and a purely datadriven DMD.

\subsection{Galerkin Projection}

Projection based methods approximate the quantity of interest as a linear combination of some spatial modes each weighted by a temporal coefficient, or

$$
\mathbf{y}(t) \approx \mathbf{U a}(t)
$$

where $\mathbf{U}$ is matrix whose columns are the spatial modes that comprise the reduced space, and a is a vector of the temporal expansion coefficients. Insertion of Eq. (2) into $d \mathbf{y} / d t=\mathbf{A}(t) \mathbf{y}$ and multiplication of the result on the left by $\mathbf{U}^{T}$ leads to the Galerkin-projected system

$$
\mathbf{U}^{T} \mathbf{U} \frac{d \mathbf{a}(t)}{d t}=\mathbf{U}^{T} \mathbf{A} \mathbf{U} \mathbf{a}(t) .
$$

Let $\mathbf{A}_{\mathbf{r}}=\mathbf{U}^{T} \mathbf{A} \mathbf{U}$, and require $\mathbf{U}$ to be orthonormal matrix (i.e, $\mathbf{U}^{T} \mathbf{U}=1$ ). Then Eq. (3) reduces to

$$
\frac{d \mathbf{a}(t)}{d t}=\mathbf{A}_{\mathbf{r}} \mathbf{a}(t)
$$

Here $\mathbf{A}_{\mathbf{r}} \in \mathbb{R}^{r \times r}$ where $r \ll n$, and it represents the low-rank approximation of $\mathbf{A}$. With this projection, the physical model is simplified to a set of $r$ coupled ODEs. Now, the problem remains to find the basis functions that adequately describe the system and result in an efficient ROM that can predict the quantities of interest accurately.

Proper orthogonal decomposition is a technique used to reveal the coherent structure in a spatiotemporal data, and it has been widely used in reduced-order modeling (see, e.g., its modern applications in Ref. [9]). First, let $\mathbf{X}=\left[\mathbf{x}_{1}, \mathbf{x}_{2}, \ldots, \mathbf{x}_{n}\right]$ be a matrix constructed from a sequence of 
temporal snapshots $\mathbf{x}_{i}$ separated in time by $\Delta t$ and produced by the FOM. The SVD of this data matrix is evaluated, or

$$
\mathbf{X}=\mathbf{U} \boldsymbol{\Sigma} \mathbf{V}^{H}
$$

where $\mathbf{U}$ is a unitary matrix whose columns are the left-singular vectors, $\boldsymbol{\Sigma}$ is a diagonal matrix of singular values, $\mathbf{V}$ consists of the right-singular vectors, and $(.)^{H}$ denotes the hermitian conjugate. Assuming the data has the potential for reduction, the first $r$ columns of $\mathbf{U}$ are used to construct a subspace onto which the system will be projected.

Dynamic mode decomposition is an alternative to POD that produces an explicit, spatio-temporal decomposition of $\mathbf{X}$. While developed as a data-driven method, we use the resulting DMD modes for the Galerkin projection. To illustrate, let $\mathbf{X}_{1}=\left[\mathbf{x}_{1}, \mathbf{x}_{2}, \ldots, \mathbf{x}_{n-1}\right]$ and $\mathbf{X}_{2}=\left[\mathbf{x}_{2}, \mathbf{x}_{2}, \ldots, \mathbf{x}_{n}\right]$. Then assume there is some A such that

$$
\mathbf{X}_{2} \approx \mathbf{A X}_{1}
$$

The problem now reduces to finding the eigenpairs of the operator A. As a first step, the POD modes need to be estimated for the matrix $\mathbf{X}_{1}$ in a way similar to what has been discussed above. By using those POD modes, an approximation of the operator $\mathbf{A}$ can be defined as

$$
\tilde{\mathbf{A}}=\mathbf{U}_{\mathbf{r}}^{\mathbf{H}} \mathbf{A} \mathbf{U}_{\mathbf{r}} \text {. }
$$

Since the matrix $\tilde{\mathbf{A}}$ is related to $\mathbf{A}$ via a similarity transformation, they have a common set of eigenvalues, which can be found by evaluating the eigenvalue decompositions, $\tilde{\mathbf{A}} \mathbf{W}=\mathbf{W} \Lambda$. The DMD modes can then be expressed as

$$
\Phi^{D M D}=\mathbf{X}_{\mathbf{2}} \mathbf{V}_{\mathbf{r}} \Sigma_{\mathbf{r}}^{-1} \mathbf{W} .
$$

These modes are then orthogonalized and used in the Galerkin projection.

\subsection{Data Driven DMD}

Data-driven DMD can be used to produce a ROM based on the same decomposition described above. Specifically, the state vector $\mathbf{x}$ can be predicted in time via

$$
\mathbf{x}^{D M D}(t)=\sum_{i=1}^{r} b_{i} \phi_{i}^{D M D} e^{w_{i} t}=\boldsymbol{\Phi}^{D M D} \operatorname{diag}\left(e^{\mathbf{w} t}\right) \mathbf{b},
$$

where $\mathbf{b}$ is the amplitude of $\mathbf{x}_{1}$ projected onto the DMD modes, and $w_{i}$ is the continuous analog of the discrete eigenvalues, i.e, $w_{i}=\log \left(\lambda_{i}\right) / \Delta t$, where $\lambda_{i}$ is the $i^{\text {th }}$ diagonal element in $\boldsymbol{\Lambda}$.

\subsection{Application and Results}

Each of the three reduced-order models techniques discussed were applied to the FOM defined in Section 2. The quantities of interest (QoIs) analyzed were the fluxes and the precursor concentrations. For the Galerkin-projection models, a basis set was generated for each group flux and each precursor group. For the POD and DMD bases, 20 modes were used. For data-driven DMD, snapshots of the QoIs were stacked into one matrix to build a DMD surrogate with 45 DMD modes. While the error for POD always decreases when more basis functions are included, this is not so for DMD. Hence, different ranks were tried, and the one producing the smallest error was used. 
Shown in Table 3 are the relative errors in the predictions of each QoI averaged over space and time. For the precursor concentrations, only the results of one group are shown, but all other groups exhibit similar errors. The group fluxes were used to compute the time-dependent core power, and the resulting errors are shown in Figure 2.

Table 3: Mean Relative Error Percentage in Responses of Interest

\begin{tabular}{c|c|c|c} 
Model & Fast flux & Thermal flux & Precursor concentration \\
\hline POD-Galerkin & $7 \times 10^{-7}$ & $7 \times 10^{-7}$ & $3.4 \times 10^{-7}$ \\
\hline DMD-Galerkin & 1.59 & 1.9 & 1.0 \\
\hline DMD & 2.66 & 2.69 & 10.40
\end{tabular}

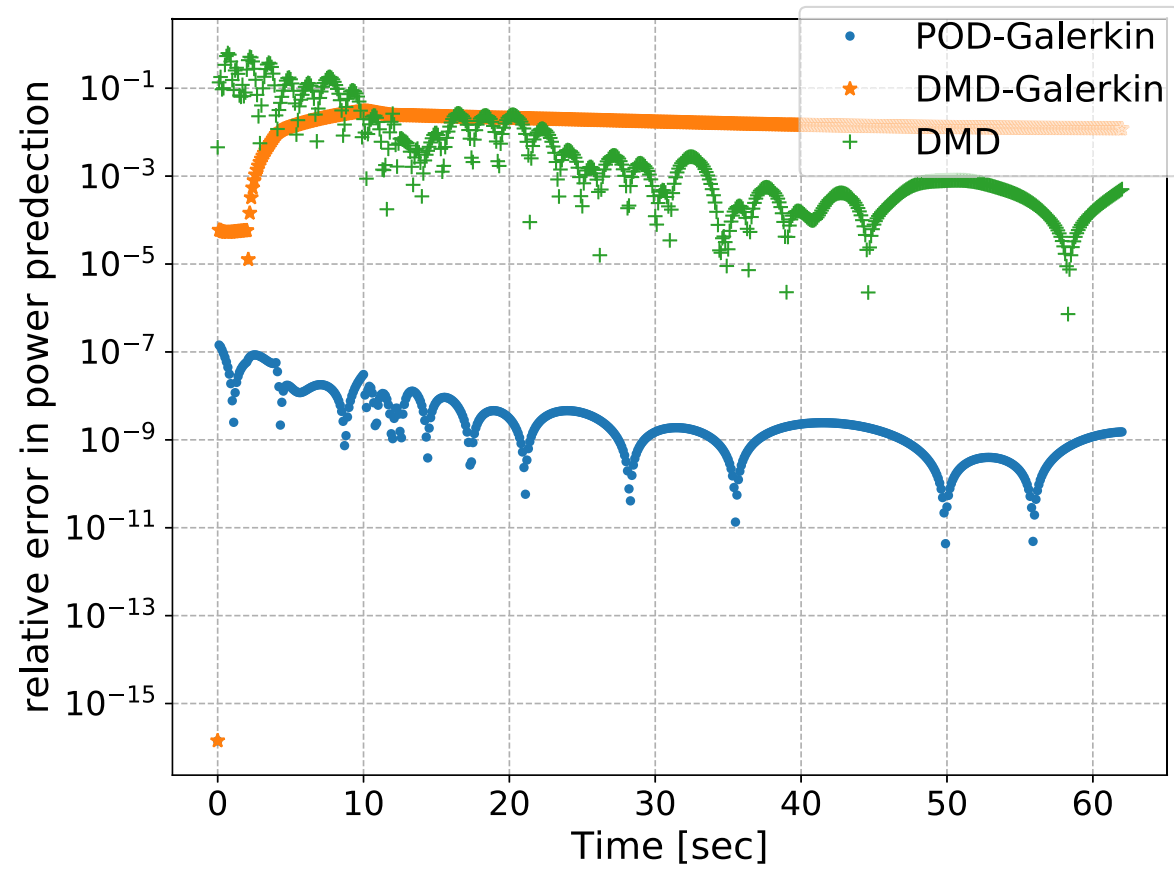

Figure 2: Relative errors in power predictions by the surrogates

As shown, the POD-Galerkin method always performs better. However, it was observed that in the case of DMD, the error in the early snapshots contributes significantly to the mean error, which suggests that future work may address this issue by using variations of the basis DMD algorithm. Since the purpose of this study is to explore different surrogates models that can serve as a computationally cheap tool to propagate uncertainties, we concluded that the POD-Galerkin method will be more efficient to be used in uncertainties propagation of the model parameters, i.e the group constants, as will be described in the next section. 


\section{Uncertainty Quantification}

While POD-Galerkin performed best for reproducing the nominal system QoI's, a ROM based on a single point in the parameter space (e.g., the nominal value) may not be accurate across the whole space. Thus, a greedy sampling method combined with POD was used to explore the parameter domain and to select those samples that best capture the domain variation.

\subsection{POD-Greedy algorithm}

The POD-Greedy algorithm combines POD with a greedy search of the parameter space. The aim is to construct a reduced space that is able to capture the whole parameter domain variation, ending up with a ROM that can be used for accurate and efficient uncertainty propagation. The PODGreedy algorithm produces a hierarchical space by incrementally enriching the reduced basis with POD modes computed from the FOM solution at a selected parameter point, which here is a vector of perturbed group constants and initial control positions. We select the parameter point at which the reduced basis yields the worst predicted error. The search terminates when the reduced space rank reaches a limit or the predicted error is below some tolerance. The reduced space is initialized with the first $r_{1}$ POD modes computed using a single random sample from a precomputed training set $P_{\text {train }}$. The premise is that with a few well-selected parameter points, we can construct a subspace that represents the whole parameter domain. The term $\Delta(\mathbf{U}, \mu)$ in Algorithm 1 measures the error in the prediction of the QoI using the current reduced subspace.

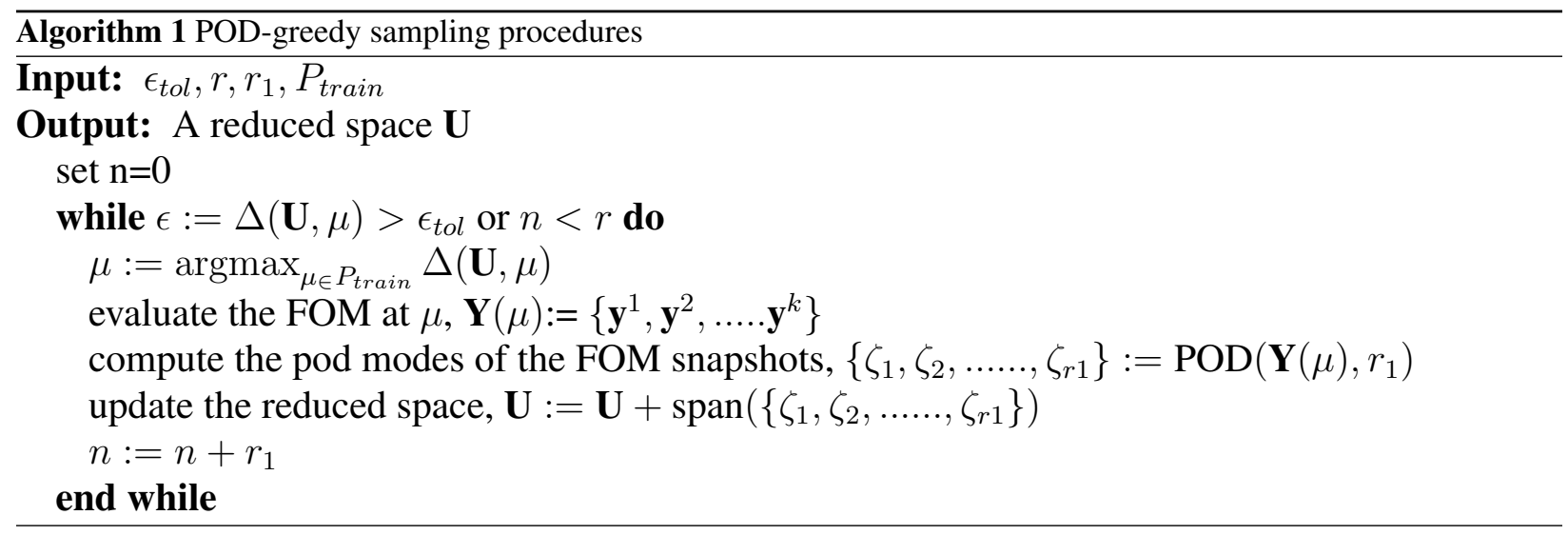

\subsection{Results}

The Greedy-POD algorithm was implemented and applied to the model problem subject to an uncertain input space. Specifically, all two group constants and initial control positions were assumed to be distributed uniformly $\pm 1 \%$ about their nominal values. The FOM was used to generate 50 training samples, of which 16 were ultimately selected by the algorithm. For each sample, the POD modes were computed. Two of these were retained at each iteration and added to the reduced space. Thus, we ended up with 32 modes for each basis set. The error was measured by using

$$
\Delta(\mathbf{U}, \mu)=\mathbf{U} \mathbf{y}^{k}-(\mathbf{I}-\mathbf{A} \Delta t) \mathbf{U} \mathbf{y}^{k+1},
$$

which estimates the error resulting from projecting the QoI, evaluated at this parameter sample, on the space of $\mathbf{U}$. The sample (of all remaining samples) for which the ROM yields the worst error 
is selected, and the reduced space is enriched by its inclusion.

To propagate uncertainties, 100 additional samples of the input parameters were generated. The FOM was executed for each, and the results were used to compute the mean and standard deviation of all QoIs. In addition, two POD-Galerkin were produced, one using data only from the nominal (mean) input parameters, i.e, single POD and one using the greedy basis. Both ROMs were executed for each of the 100 samples, and the outputs were again used to estimate the mean and standard deviation of all QoIs. Shown in Table 4 are the maximum relative errors (\%) in the mean and standard deviation over all cells and the precursors groups. Figure 3 shows the relative error in the predicted mean and standard deviation of the time dependent power derived from the twogroup flux. As can be seen, the POD-Greedy algorithm gives very promising results, although the
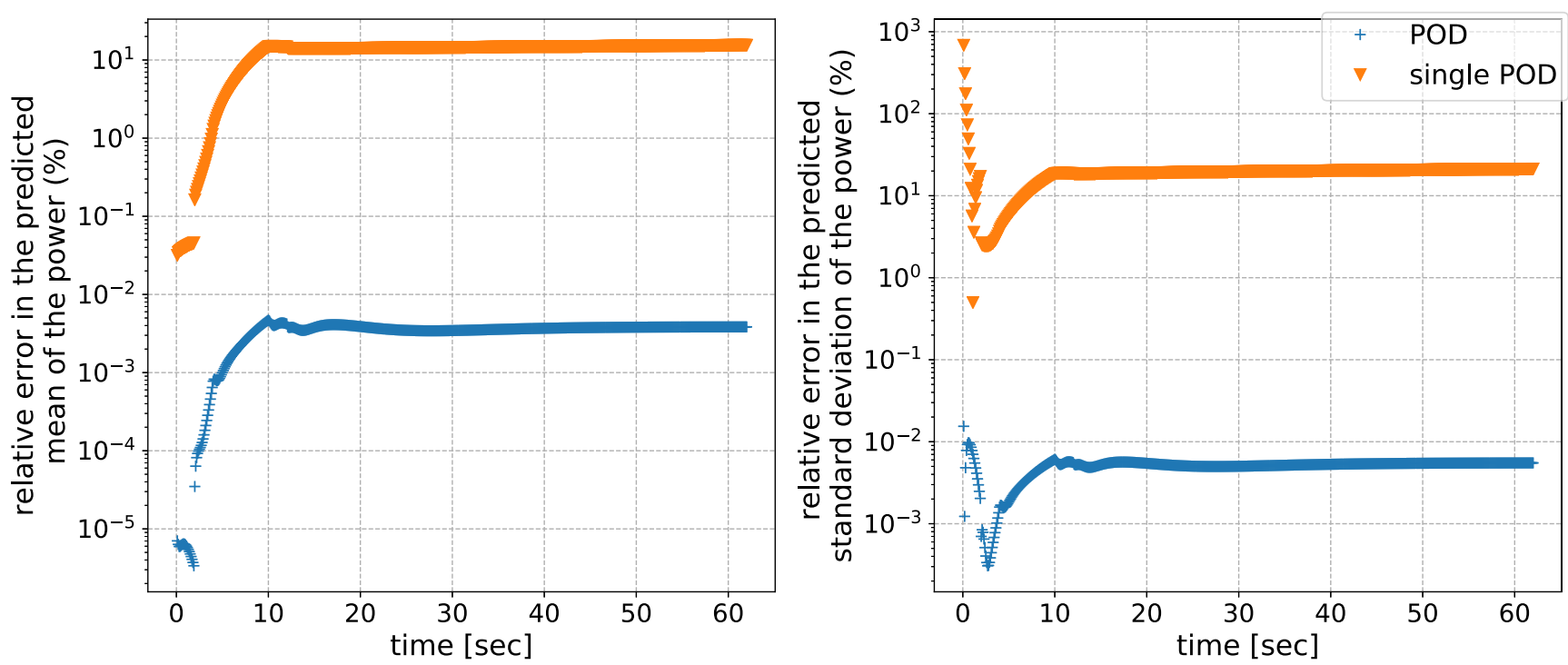

Figure 3: Relative errors in power mean and standard deviation prediction

variance in the uncertain space is meaningful as can be implied from the results of the single POD where the basis is coming from the middle point in the space.

Table 4: the maximum relative errors $(\%)$ in the mean (standard deviation)

\begin{tabular}{l|ccc}
\hline & $\begin{array}{c}\text { Thermal flux } \\
(\text { cell 5) }\end{array}$ & $\begin{array}{c}\text { Fast flux } \\
(\text { cell 5) }\end{array}$ & $\begin{array}{c}\text { Precursor concentration } \\
\text { (group 7, cell 1) }\end{array}$ \\
\hline Greedy-POD & $0.00472(0.00612)$ & $0.00472(0.00611)$ & $0.0378(0.1333)$ \\
Single POD & $15.49695(21.2416)$ & $15.5059(31.133)$ & $13.33(49.379)$
\end{tabular}




\section{Conclusions}

The potential of different ROMs to approximate a neutronic transient model was evaluated. The intrusive POD-Galerkin and DMD-Galerkin were shown to outperform the non-intrusive, datadriven DMD. Of the three ROMs, POD-Galerkin performed best, with maximum, relative errors on the order of $\approx 10^{-7}$ for the group fluxes. The POD-Galerkin model was used to propagate uncertainties in the group constants and the initial control position to uncertainties in the group fluxes and the precursors concentrations. Greedy sampling was used to construct an optimum reduced space for use with the POD-Galerkin procedure. With this subspace, the ROM was able to predict the mean and the standard deviation of the QoIs with a good accuracy. In particular, the maximum relative errors in the predicted mean and the standard deviation of the time dependent power were $0.00469 \%$ and $0.0154 \%$, respectively. Although intrusive techniques are often hard to implement in practice, it is believed that the methods described here can relatively easily be implemented within a variety of finite-element models under development at national laboratories and elsewhere.

\section{ACKNOWLEDGEMENTS}

The content presented is based on work supported by the US Department of Energy Office of Nuclear Energy Contracts DE-NE0008408 and DE-NE0008750.

\section{REFERENCES}

[1] M. Abdo, R. Elzohery, and J. A. Roberts. "Modeling isotopic evolution with surrogates based on dynamic mode decomposition." Annals of Nuclear Energy, volume 129, pp. 280-288 (2019).

[2] R. Elzohery, M. Abdo, and J. Roberts. "Comparison Between Gaussian Processes and DMD Surrogates for Isotopic Composition Prediction." Transactions of the American Nuclear Society, volume 118, pp. 459-462 (2018).

[3] X. Wu, C. Wang, and T. Kozlowski. "Kriging-based Surrogate Models for Uncertainty Quantification and Sensitivity Analysis." (2017).

[4] Z. K. Hardy, J. E. Morel, and C. Ahrens. "Dynamic Mode Decomposition for Subcritical Metal Systems." Nuclear Science and Engineering, pp. 1-13 (2019).

[5] S. Lorenzi. "An Adjoint Proper Orthogonal Decomposition method for a neutronics reduced order model." Annals of Nuclear Energy, volume 114, pp. 245-258 (2018).

[6] S. Lorenzi, A. Cammi, L. Luzzi, and G. Rozza. "A reduced order model for investigating the dynamics of the Gen-IV LFR coolant pool." Applied Mathematical Modelling, volume 46, pp. 263-284 (2017).

[7] A. Quarteroni, G. Rozza, and A. Manzoni. "Certified reduced basis approximation for parametrized partial differential equations and applications." Journal of Mathematics in Industry, volume 1(1), p. 3 (2011).

[8] P. Chen, A. Quarteroni, and G. Rozza. "Reduced order methods for uncertainty quantification problems." ETH Zurich, SAM Report, volume 3 (2015).

[9] P. Benner, A. Cohen, M. Ohlberger, and K. Willcox. Model reduction and approximation: theory and algorithms, volume 15. SIAM (2017). 